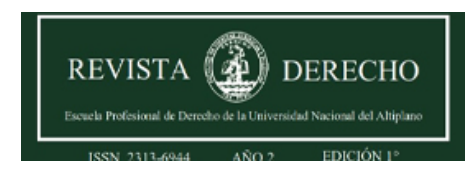

Revista de Derecho

ISSN: 2313-6944

ISSN: 2707-9651

revistaderecho@unap.edu.pe

Universidad Nacional del Altiplano

Perú

\title{
LA OTRA PANDEMIA: VIOLACIÓN DE LA LIBERTAD SEXUAL DE MENORES EN UN CONTEXTO INTRA FAMILIAR, ANÁLISIS CRIMINOLÓGICO ANTE EL INCREMENTO DE CASOS DURANTE EL CONFINAMIENTO POR EL COVID-19
}

ESPINOZA ROCHA, Frank Michael; VELASCO VEGA, Vanesa Valeria; ACERO LEON, Eddy

LA OTRA PANDEMIA: VIOLACION DE LA LIBERTAD SEXUAL DE MENORES EN UN CONTEXTO INTRA

FAMILIAR, ANÁLISIS CRIMINOLÓGICO ANTE EL INCREMENTO DE CASOS DURANTE EL CONFINAMIENTO POR EL COVID-19

Revista de Derecho, vol. 6, núm. 1, 2021

Universidad Nacional del Altiplano, Perú

Disponible en: https://www.redalyc.org/articulo.oa?id=671870928005

DOI: https://doi.org/10.47712/rd.2021.v6i1.110

\section{(c) ()}

Esta obra está bajo una Licencia Creative Commons Atribución 4.0 Internacional. 
Artículos de investigaciones realizadas por estudiantes con docentes

\section{LA OTRA PANDEMIA: VIOLACIÓN DE LA LIBERTAD SEXUAL DE MENORES EN UN CONTEXTO INTRA FAMILIAR, ANÁLISIS CRIMINOLÓGICO ANTE EL INCREMENTO DE CASOS DURANTE EL CONFINAMIENTO POR EL COVID-19}

\section{THE OTHER PANDEMIC: VIOLATION OF THE SEXUAL FREEDOM OF MINORS IN AN INTRA FAMILY CONTEXT, CRIMINOLOGICAL ANALYSIS FACING THE INCREASE OF CASES DURING THE CONFINEMENT BY COVID-19}

Frank Michael ESPINOZA ROCHA

Universidad Nacional del Altiplano, Perú

espinozafrank90@gmail.com

Vanesa Valeria VELASCO VEGA

Universidad Nacional del Altiplano, Perú

ing.valeriavelasco@hotmail.com

Eddy ACERO LEON

Universidad Nacional del Altiplano de Puno, Perú

alt24_416@hotmail.com
DOI: https://doi.org/10.47712/rd.2021.v6i1.110 Redalyc: https://www.redalyc.org/articulo.oa? $\mathrm{id}=671870928005$

Recepción: 09 Octubre 2020

Aprobación: 07 Enero 2021

Publicación: 08 Enero 2021

\section{Resumen:}

El presente trabajo tiene como finalidad analizar desde el enfoque criminológico la otro pandemia que no tiene la suficiente atención y preocupación por parte del estado y la ciudadanía, la violencia de la libertad sexual contra menores, en nuestro contexto de la pandemia por el virus Covid-19 para lo cual acudimos a fuentes estadísticas, conceptuales, doctrinales ,además de un análisis criminológico crítico, lo que nos permitió primero contextualizar cual era la situación en nuestro país antes de la pandemia y durante la pandemia, analizar el actuar de nuestro Estado a través de instituciones como el Ministerio de la Mujer y Poblaciones Vulnerables , programas como el centro de ayuda mujer y las ahora implementados instrumentos línea 100 y 1801 , para así poder concluir cuales son las teorías criminológicas de la conducta criminal que establece los factores criminológicos por los que se presentaba este acto delictivo y por los que se incrementó durante el confinamiento.

Palabras Clave: Violencia de la libertad sexual, menores de edad, Pandemia -Covid19, factores criminológicos, conducta criminal.

\section{AbstraCt:}

The purpose of this work is to analyze from the criminological approach the other pandemic that does not have enough attention and concern on the part of the state and citizens, the violence of sexual freedom against minors in our context of the COVID 19 pandemic, for which we go to statistical, conceptual and doctrinal sources in addition to a critical criminological analysis, which allowed us to first contextualize what was the situation in our country before the pandemic and during the pandemic, analyze the actions of our State through institutions such as the Ministry of the Women and Vulnerable Populations, programs such as the women's help center and the now implemented instruments line 100 and 1801, in order to conclude which are the criminolgical theories of criminal conduct that establish the criminological factors for which this criminal act was presented and why those that increased during confinement.

KEYWORDS: Violence of sexual freedom, minors, Pandemic -Covid19, criminological factors, criminal conduct.

\section{Notas DE AUTOR}

espinozafrank90@gmail.com 


\section{INTRODUCCIÓN}

Las cifras de violencia de libertad sexual incrementaron en todo el mundo, por el confinamiento media que se adoptó para evitar la propagación del Covid-19 y en nuestro país no es ajeno a esta situación, tomándose esta medida preventiva como la principal arma de combate, mediante Decreto Supremo No 044-2020-PCM en fecha 15 de marzo 2020 sin embargo no se previó las consecuencias del confinamiento, esto hizo que se reforzara la situación de aislamiento en la que se encuentran miles de menores que conviven con su agresor adicionalmente ha provocado que muchos de ellos tengan menos acceso a los recursos de protección( poder contar la situación que pasan a otros familiares, denuncias o llamadas de alerta). Además, se observa que la crisis económica actual provoco que las personas agresoras sufran de frustraciones, estrés, que afloren sus taras emocionales, aumente su agresividad y estos sean canalizados en el miembro familiar más cercano y vulnerable, los niños y mujeres.

Según se recoge en un artículo publicado por ONU, la enfermedad por el Covid-19 se ha convertido en la "situación perfecta" para ejercer un comportamiento controlador y violento en el hogar. Y el aumento de las cifras en nuestro país lo confirman. En nuestro País se ha intentado frenar esta "otra pandemia" implementando la línea 100 para que ya las víctimas o algún familiar, vecino testigo de la agresión pueda denunciar ante las autoridades sin embargo a pesar de esto solo durante los primeros 6 meses del 2020 ya se superó el total de los casos producidos durante el mismo periodo de tiempo del año 2019.

La violencia de la libertad sexual es una de las principales causas de sufrimiento para los menores y las familias, teniendo consecuencias a largo plazo. La agresión causa estrés y se asocia a trastornos del desarrollo cerebral temprano. Los casos extremos de estrés pueden alterar el desarrollo de los sistemas nervioso e inmunitario. En consecuencia, los adultos que han sufrido este tipo de violencia en la infancia corren mayor riesgo de sufrir problemas conductuales, físicos y mentales además de repetir la agresión volviéndose en un círculo vicioso.

Teniendo como principales objetivos analizar la situación pre-pandemia y actual de los casos de abuso sexual a menores, para así poder determinar si se evidencia un incremento de casos producto del confinamiento por el virus Covid-19, como segundo objetivo desde la criminología aplicada y clínica identificar los factores criminológicos que coadyuvan a que se produzca este ilícito penal, finalmente analizaremos el actuar y respuesta del Estado frente a este problema social durante la pandemia.

\section{VIOLENCIA SEXUAL}

Según el Observatorio Nacional de la Violencia Contra las Mujeres y los Integrantes del Grupo Familiar del Ministerio de la Mujer y Poblaciones Vulnerables (MIMP), la violencia sexual comprende todas las acciones con naturaleza sexual que se cometen contra una persona sin su consentimiento o bajo coacción. Esto incluye actos que no involucran penetración o contacto físico así como todo acto que vulnera el derecho de las personas a decidir voluntariamente sobre su vida sexual o reproductiva, a través de amenazas, coerción o intimidación.

La Organización Mundial de la Salud -OMS define la violencia sexual como: "todo acto sexual, la tentativa de consumar un acto sexual, los comentarios o insinuaciones sexuales no deseados, o las acciones para comercializar o utilizar de cualquier otro modo la sexualidad de una persona mediante coacción por otra persona, independientemente de la relación de esta con la víctima, en cualquier ámbito, incluidos el hogar y el lugar de trabajo".

La coacción puede abarcar:

- Uso de grados variables de fuerza 
- Intimidación psicológica

- Extorsión

- Amenazas (por ejemplo de daño físico o de no daño hacia un familiar, etc.)

También puede haber violencia sexual si la persona no está en condiciones de dar su consentimiento, por ejemplo cuando está ebria, bajo los efectos de un estupefaciente, dormida o mentalmente incapacitada.

La definición de la OMS es muy amplia, pero también existen definiciones más circunscritas. Por ejemplo, para fines de investigación algunas definiciones de violencia sexual se limitan a los actos que incluyen la fuerza o la amenaza de violencia física.

\subsection{VIOLENCIA SEXUAL CONTRA MENORES}

UNICEF define la violencia sexual contra los niños como una grave violación de sus derechos. Sin embargo, es una realidad en todos los países y grupos sociales. Toma la forma de abuso sexual, acoso, violación o explotación sexual en la prostitución o la pornografía. Puede ocurrir en los hogares, instituciones, escuelas, lugares de trabajo, en las instalaciones dedicadas al viaje y al turismo, dentro de las comunidades, en contextos de desarrollo y de emergencia.

Para mejor estudio el Ministerio de Salud ha venido implementando los Módulos de Atención al Maltrato Infantil y del Adolescente en Salud, para la prevención e intervención del maltrato infantil-adolescente, en los niños y niñas desde los 0 años hasta los 11 años, 11 meses y 29 días y, los adolescentes desde los 12 años a 17 años, 11 meses y 29 días.

La verdadera magnitud de la violencia sexual está oculta, debido a su naturaleza sensible e ilegal. La mayoría de los niños y las familias no denuncian los casos de abuso y explotación a causa del estigma, el miedo y la falta de confianza en las autoridades. La tolerancia social y la falta de conciencia también contribuyen que no se denuncien muchos de los casos.

a. TIPOS DE AGRESIÓN SEXUAL POR LA DISTANCIA RELACIONAL CONTRA MENORES

Se distingue dos tipos de abuso sexual a partir del análisis de la distancia relacional entre niño, niña y abusador. Uno de ellos es el abuso sexual extra familiar, que ocurre cuando el agresor no pertenece al medio familiar, pudiendo ser un sujeto totalmente desconocido del niño, la niña y su familia, o algún conocido que pertenezca a su entorno. Durante el confinamiento y según declaraciones de la ministra del MIMP en el 20\% de casos los victimarios son vecinos que quedan a cargo del cuidado del menor debido a los familiares del menor se vieron obligados a salir a trabajar. En este último tipo, el abusador goza sometiendo a su víctima por la fuerza o el terror y habitualmente es un hecho único, muy violento, por lo que, en este tipo de abuso, los niños o niñas se reconocen más fácilmente como víctimas e identifican al adulto como su agresor.

a.1 Violencia intrafamiliar

Otro tipo de abuso sexual es el intrafamiliar, que se refiere al contacto sexual entre un niño o niña y un familiar consanguíneo de la unidad familiar primaria nuclear (padre, hermanos, abuelos, tíos, padrastros y hermanastros). En este caso, la agresión presenta características distintivas, relacionadas con el hecho de que el agresor manipula el vínculo familiar, a través del poder que le confiere su rol. Generalmente, es una agresión reiterada en el tiempo, en la que se impone la dinámica del secreto y su revelación resulta tardía. Suele darse en familias disfuncionales y son el resultado de múltiples factores que bloquean o perturban los mecanismos naturales que regulan la sexualidad al interior de la familia.

Es por esto que la mayoría de los abusos sexuales contra los niños y adolescentes, en tales casos, se producen en el hogar. E incluye madrastras, padrastros, tutores, hermanastros, abuelos e incluso novios o parejas que viven con el padre o la madre y asumen el papel de cuidadores. La familiaridad entre el niño y el abusador presenta fuertes lazos emocionales, tanto positivos como negativos, lo que contribuye a que en este tipo de abuso ocurra un mayor impacto cognitivo-comportamental para el niño y su familia. Otro factor importante que puede surgir en esta difusa relación conyugal, es el miedo o la dependencia material que pueden llevar a la esposa a aceptar la situación. 


\section{SITUACIÓN EN EL PERÚ}

\subsection{ANTES DE LA PANDEMIA}

Según la Defensoría del Pueblo en su reporte principales resultados de la supervisión a la atención de niños, niñas y adolescentes víctimas de violencia en los módulos de atención al maltrato infantil y del adolescentes en salud. MAMIS. Conjuntamente con el Ministerio de la Mujer y Poblaciones Vulnerables, años 2017, 2018,2019 .

TABLA No 01

\begin{tabular}{|l|r|r|r|}
\hline \multicolumn{4}{|c|}{ CASOS DE VIOLENCIA CONTRA NIÑOS, NIÑAS Y ADOLESCENTES - CEM } \\
\hline \multicolumn{1}{|c|}{ AÑO } & 2017 & 2018 & 2019 \\
\hline Violencia física & 10142 & 13752 & 12733 \\
\hline Violencia psicológica & 13830 & 18911 & 18617 \\
\hline Violencia económica & 116 & 189 & 246 \\
\hline violencia sexual & 6593 & 8957 & 8608 \\
\hline Total & 30681 & 41809 & 40204 \\
\hline \hline
\end{tabular}

Defensoría del Pueblo (2019)

El cuadro superior nos señala que durante el 2017 los centros de Emergencia Mujer (CEM) atendieron 30681 casos de los cuales 6593 son de violencia sexual, respectivamente en el año 2018 existieron 41809 casos de violencia de los cuales 8957 fueron de violencia sexual, en el 2019 se presentaron 40204 casos y de los cuales 8608 fueron de violencia sexual.

Como se advierte en los últimos años, los casos de violencia contra la niñez y adolescencia en el Perú, están siendo denunciados con mayor énfasis, lo que exige que el Estado brinde una respuesta integral de manera inmediata; sobre todo en las regiones con mayor índice de registro de atención de casos en los CEM. De acuerdo a lo informado por el Ministerio de la Mujer y Poblaciones Vulnerables, las diez regiones que reportaron más casos de violencia contra niños, niñas y adolescentes son: Lima, Arequipa, Cusco, Junín, La Libertad, Ancash, San Martín, Callao, Ica y Piura.

Cabe indicar que, el artículo 21 del Código de los Niños y Adolescentes establece que el niño, la niña y adolescente tienen derecho a la atención integral de salud; asimismo, su artículo $38^{\circ}$ dispone que quienes son víctima de maltrato físico, psicológico o de violencia sexual merecen que se les brinde atención integral mediante programas que promuevan su recuperación física y psicológica. Dicho servicio debe estar a cargo del Sector Salud y los programas deberán incluir a la familia.

En el informe estadístico de setiembre del 2019 del Instituto Nacional Penitenciario, las violaciones sexuales y los actos contra el pudor se encuentran entre los delitos específicos más comunes del Perú. 


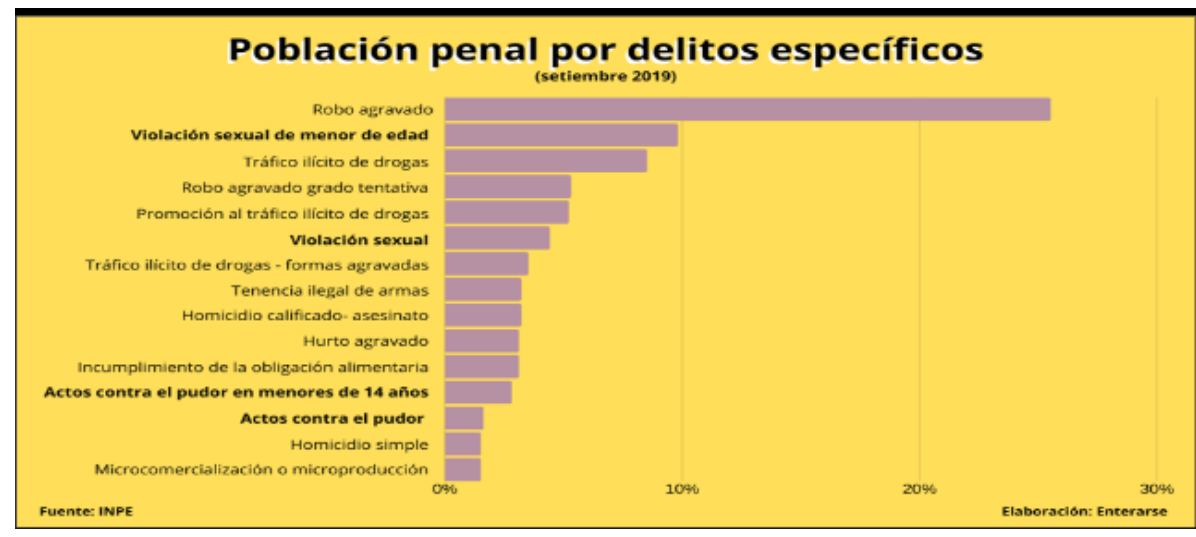

FIGURA No01

Población Penal Por Delitos Específicos.

Enterarse, 2019

En la figura $\mathrm{N}^{\circ} 1$ observamos que el $9.8 \%$ de la población penitenciaria está presa por violación sexual a menores y es la segunda causa de encarcelamiento más común después del robo agravado. Asimismo, el 4.4\% de los reos se encuentra preso por violación sexual, el $2.8 \%$ por actos contra pudor contra menores de 14 años y el $1.6 \%$ por actos contra el pudor.

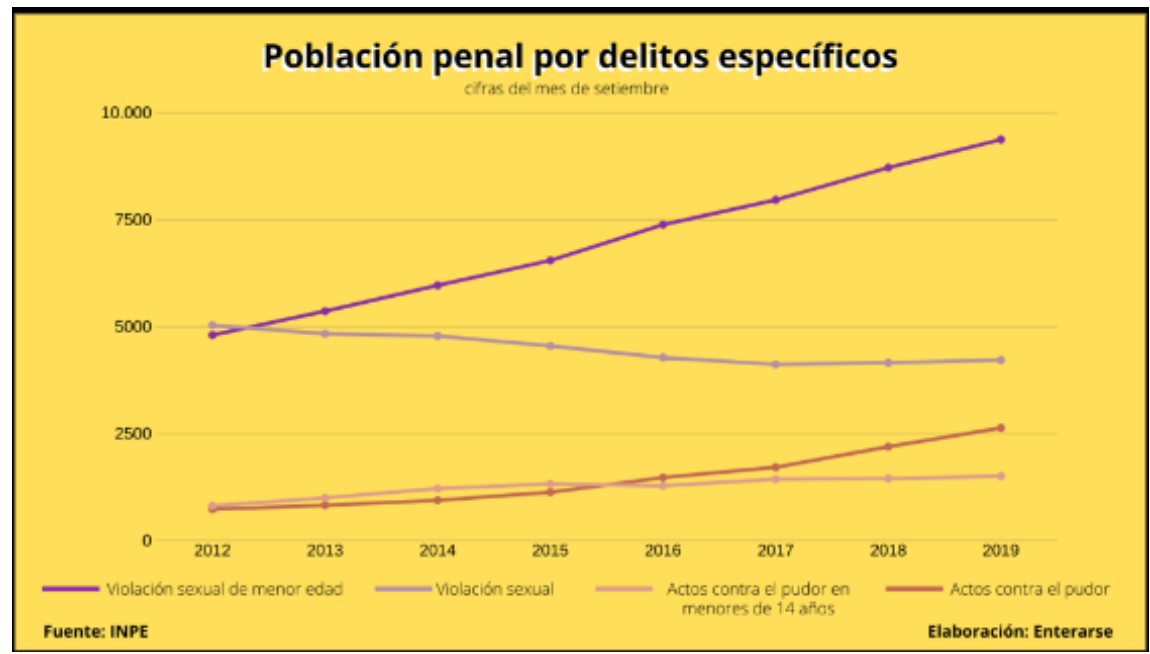

FIGURA $\mathrm{N}^{\circ} 02$

Población Penal Por Delitos Específicos Mes De Setiembre Del 2012 Al 2019

Enterarse, 2019

En la figura $\mathrm{N}^{\circ} 2$ se observa que desde setiembre del 2012 a setiembre del 2019, la población penal por delitos relacionados a violencia sexual ha aumentado.

No obstante, resalta el incremento del número de personas por el delito de violación sexual de menores de edad. En 8 años, ha aumentado en un 95\%, siendo el delito de índole sexual con la segunda tasa de crecimiento más alta. Este porcentaje es solamente superado por el crecimiento del número de reos por actos contra el pudor contra menores de 14 años, que ha sido de un 118\% en el mismo periodo de tiempo.

Ahora, si tomamos en cuenta el crecimiento de la población penitenciaria, esta ha crecido en menor medida que el número de presos por el delito de violación sexual a menores y actos contra el pudor a menores de 14 años.

Antes de ver el número de denuncias por violencia sexual, es importante resaltar el incremento del número de los Centro Emergencia Mujer (CEM) porque esto hace más accesible el proceso de atención y denuncia. 
En el 2012 había 175 CEM, mientras que, para setiembre del 2019, la cifra aumentó a 383. Desde el 2018, la cifra ha aumentado en 36 .

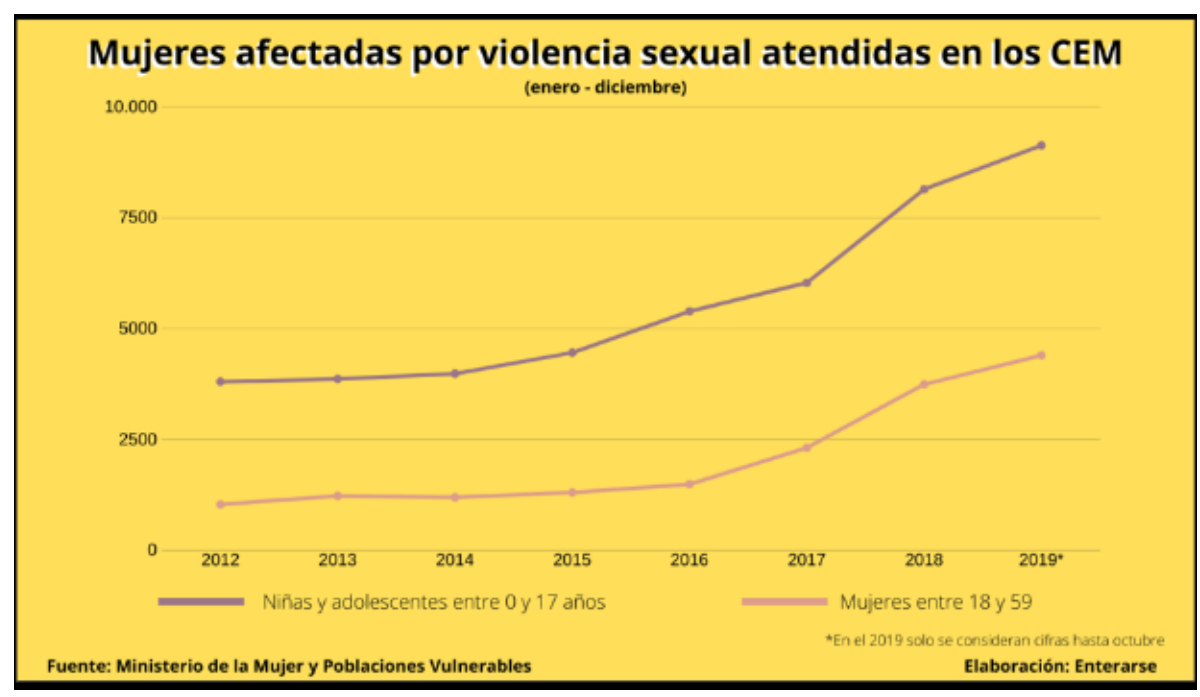

FIGURA $\mathrm{N}^{\circ} 03$

Mujeres Afectadas por Violencia Sexual MIMP, 2019.

Del total de número de casos atendidos, 3,800 fueron niñas y adolescentes.Para el 2019, esta cifra aumentó a 10,520 menores entre 0 y 17 años atendidos en los CEM. Para el 2019, sin embargo, solo se han considerado los meses de enero a octubre, razón por la cual la cifra podría ser mayor.

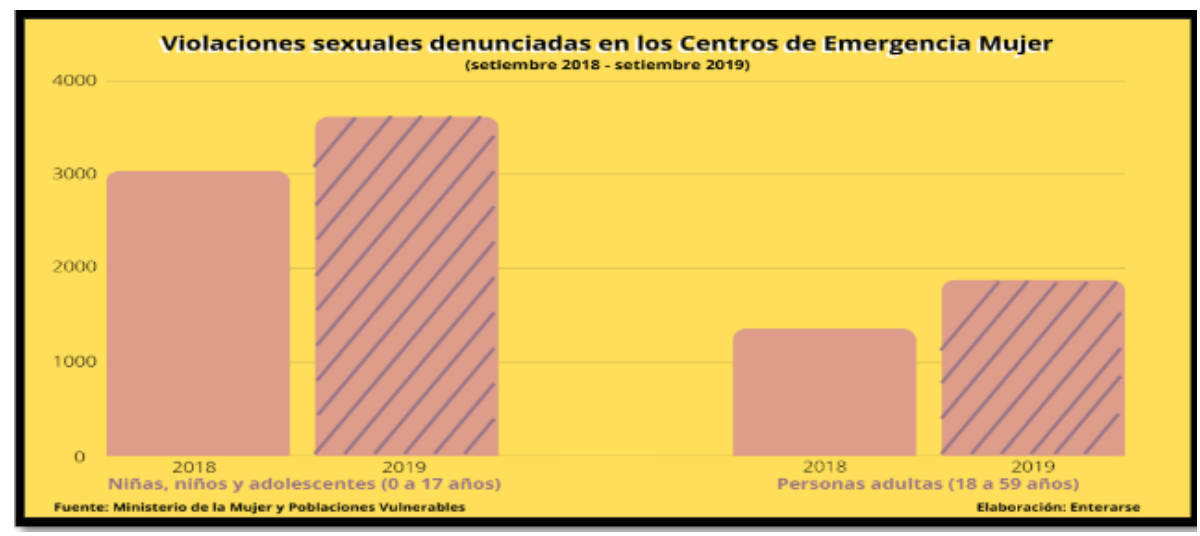

FIGURA No 04

Violencia Sexual Denunciadas

MIMP, 2019

Si vemos solo las cifras de violaciones sexuales, podemos ver que de setiembre del 2018 a setiembre del 2019 el número de denuncias por violación sexual a niñas, niños y adolescentes aumentó en 577 . Es decir, se presentó 577 casos más este año comparado con el anterior. Ahora, veamos las cifras desagregadas según departamento. Hasta setiembre del 2019, los departamentos que tuvieron más casos de violación sexual en niñas, niños y adolescentes fueron Lima (891 casos), Arequipa (283), Junín (248), La Libertad (223) y Cusco (186). En el caso de violación sexual en personas adultas, en el mismo periodo de tiempo, los cinco departamentos con mayor número de casos fueron Lima (666 casos), Arequipa (140), La Libertad (111), Junín (100) y Cusco (98). Los departamentos con mayor incidencia de casos de violación sexual se repiten en ambos grupos etarios. 
Es importante resaltar que no podemos saber con certeza hasta qué punto el aumento en el número de casos de violencia sexual denunciados en los CEM puede explicarse por el aumento de estos servicios públicos, por una mayor cultura de denuncia o porque la violencia sexual en general ha aumentado en este periodo, o tal vez los canales de prevención y/o erradicación no están siento efectivos.

Durante el año 2020 hasta antes de la declaratoria de estado de emergencia por el covid-19 los datos recolectados por el MIMP son los siguientes:

\section{CASOS DE VIOLENCIA SEXUAL HACIA NIÑAS, NIÑOS Y ADOLESCENTES ATENDIDOS POR EL PROGRAMA AURORA, según tipo de violencia ( periodo enero-marzo 2020)}

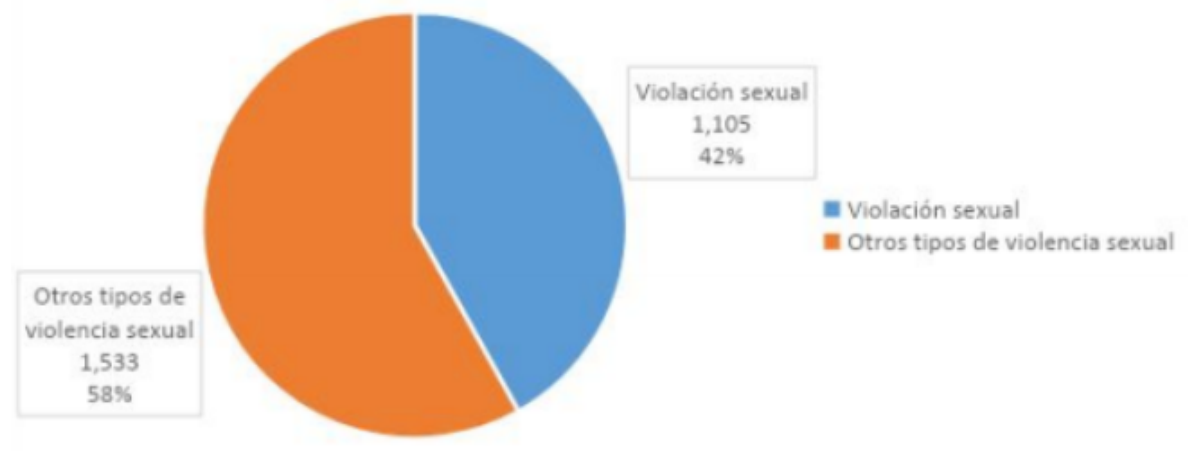

FIGURA N ${ }^{\circ} 05$

Casos de violencia sexual atendidos

Elaborado por Defensoría del Pueblo Boletín MIMP 2020

Del gráfico anterior, se advierte que, del mes de enero a marzo del presente año, los servicios del Programa AURORA atendieron un total de 1,105 casos de violación sexual contra niñas, niños y adolescente, cifra que representaría el $42 \%$ de los casos de violencia sexual que sufre este grupo. De esta cifra, se identificó que el 92\% fueron contra niñas y adolescentes. Es decir, de los 1,105 casos reportados, 1,018 fueron de niñas y adolescentes mujeres. Ello demuestra una vez más que existe un componente de género en este tipo de violencia.

\section{ANALISIS CRIMINOLOGICO DE LA VIOLACIÓN SEXUAL DE LOS MENORES DE EDAD DURANTE LA PANDEMIA}

\subsection{SITUACIÓN DURANTE LA PANDEMIA}

Durante el contexto de emergencia nacional, uno de los problemas sociales que se ha agudizado es la violencia ejercida hacia niñas, niños y adolescentes, "cuya situación de vulnerabilidad se incrementa por el aislamiento social obligatorio, así como por factores como el género, la condición socioeconómica, la discapacidad, la pertenencia a un grupo étnico, entre otros". (Redacción de la Opinión, 2020)

$\mathrm{Al}$ respecto, la Defensoría del Pueblo ha expresado, de forma reiterada, su preocupación por la afectación a la vida e integridad de niñas, niños y adolescentes, especialmente durante el confinamiento dispuesto para fines de prevención del contagio, debido a la convivencia con el/la agresor/a y las dificultades de dicho grupo poblacional para acceder a los servicios de denuncia y/o protección integral. (Defensoria del Pueblo, 2020)

En efecto el Ministerio de la Mujer y Poblaciones Vulnerables (MIMP) informo que han registrado alrededor de 226 abusos sexuales contra menores de edad desde el inicio de la pandemia el pasado 15 de marzo hasta el mes de Mayo. (Sociedad LR, 2020) 
En últimas declaraciones la ex ministra Gloria Montenegro Figueroa de esta cartera informo que hasta el mes de agosto se presentaron 513 casos de abuso sexual finalizando junio en contra de menores siendo en mayor porcentaje niñas las víctimas.

Como ultima data este número incremento exponencialmente en especial en zonas rurales, ya que en declaraciones a un medio periodístico la ministra de esta cartera informo que existían 500 casos de niñas indígenas que fueron agredidas sexualmente.

Es importante resaltar que no existe una data cierta solo aproximaciones.

3.2. ANÁLISIS DE LOS FACTORES CRIMINOLÓGICOS DE LA VIOLACIÓN SEXUAL DE MENORES DESDE LA CRIMINOLOGÍA APLICADA

La Criminología Aplicada profundiza en la naturaleza, el alcance y las causas de la delincuencia y las nuevas formas de criminalidad, así como de los métodos utilizados para detectar, contrarrestar y controlar el comportamiento criminal, poniendo énfasis en el impacto que la delincuencia produce en la comunidad social y en el sistema de justicia penal. Para la cual resaltamos dos teorías Dinámicas que nos coadyuvaran en analizar los factores principales que llevan a establecer hipótesis por las que incrementaron los casos de abuso sexual durante la pandemia: Teoría del Desarrollo y la Teoría del control social informal.

\subsubsection{TEORÍA DEL DESARROLLO}

Desde esta perspectiva se destaca el carácter abierto e inacabado de cada persona en relación con su entorno. Refiere que esta flexibilidad de las conductas persiste a lo largo de toda la vida. Esta teoría recoge del enfoque estático las diferencias individuales y la crianza de inicio temprano a la hora de explicar la conducta criminal, aun cuando su explicación principal de la criminalidad posterior se basa en los cambios que se dan a lo largo de la vida en el ámbito social de la persona. Acá se subsuman las teorías de las etapas vitales, cuya tesis nuclear menciona que al igual que los individuos cambian y maduran, también lo hacen los factores que intervienen sobre su conducta. (Thornberry \& Krohn, 2001)

En base de esta teoría establecemos que existen Factores individuales y relacionales, Factores Comunitarios y sociales, que coadyuva que estando dentro de una situación de confinamiento por la pandemia del Covid 19 genera que se consuma el delito de violación sexual de menores de edad. Las cuales desarrollaremos a continuación:

3.2.1.1. Factores individuales y relacionales

La investigación de los factores que aumentan el riesgo de que los hombres cometan actos de violencia sexual durante el confinamiento por el Covid-19 y se refiere predominantemente a hombres que fueron aprehendidos, en particular por haber cometido una violación. Entre los factores que han sido señalados en múltiples estudios de ese tipo se cuentan los siguientes:

- Pertenencia a una pandilla,

- Consumo perjudicial o ilícito de alcohol o drogas,

- Personalidad antisocial

- Exposición en la niñez a la violencia entre los padres,

- Antecedentes de abuso físico o sexual en la niñez ,lo que causa un círculo vicioso pasando de victimas a victimarios

- Escasa educación,

- Aceptación de la violencia (por ejemplo, creer que sea aceptable golpear a la Esposa o la novia),

- Opiniones favorables a la inequidad de género.

3.2.1.2. Factores comunitarios y sociales

Desde una perspectiva de salud pública, los factores comunitarios y sociales pueden ser los más importantes para identificar formas de prevenir la violencia sexual antes de que se produzca, ya que la sociedad y la cultura pueden apoyar y perpetuar creencias que aprueban la violencia. Los factores vinculados con tasas más elevadas de violencia sexual perpetrada por hombres incluyen los siguientes: 
- Normas tradicionales y sociales favorables a la superioridad masculina (por ejemplo, considerar que las relaciones sexuales sean un derecho del hombre en el matrimonio, que las mujeres sean responsables de mantener bajo control los deseos sexuales de los hombres o que la violación sea un signo de masculinidad);

- Aceptación social, muchas niñas y niños crecen escuchando noticias sobre estos casos tan a menudo, que crea en ellos un tipo de aceptación y normalización.

- Incorrecta o hasta inexistente forma de abordar temas de educación sexual de los padres con los menores, esto impide que el menor sepa reconocer o denunciar lo que le está pasando además que no se crean lazos de confianza dificultando aún más que el menor pueda comunicar lo que le está pasando.

- No darle la credibilidad a las confesiones de las víctimas, muchos menores logran denunciar sin embargo no les creen o si lo hacen, la persona adulta con el conocimiento se niega a comunicar a las autoridades debido la relación de dependencia económica aún más agravada durante la pandemia y emocional con los victimarios.

\subsubsection{LA TEORÍA DEL CONTROL SOCIAL INFORMAL.}

Se desarrolló la teoría del control social informal. Para ellos, la pregunta claves cuáles son los factores que afectan la trayectoria criminal de los individuos. Aquí, la conducta criminal se explica por la falta de controles sociales informales, y que estos varían a lo largo de la vida. (Laub \& Sampson, 2003)" Las transiciones importantes que se dan a lo largo del curso de la vida producen cambios en el nivel de control informal y pueden actuar como puntos de inflexión para el crimen" (Sampson y Laub, 1993). En estos autores, la noción de punto de inflexión cobra mayor relevancia desde sus últimas publicaciones, ya que se centran en estos para explicar el proceso que permite a las personas exhibir conductas delictivas o desistir de ellas.

Mencionan que algunos de estos puntos son el matrimonio y el apego a un cónyuge, unirse al ejército, ser enviado a la escuela de reforma y el cambio de barrio o residencia. En sentido paralelo, los individuos pueden usar estos puntos de inflexión como oportunidades para un comportamiento antisocial o delictivo, por ejemplo, el matrimonio y la violencia doméstica o el empleo y el robo en el lugar de trabajo. Así, establecimos que existen dos grandes grupos de factores que contribuyeron a la conducta criminal de los agresores de menores de edad: Fronteras Culturales y Factor económico.

3.2.2.1. Fronteras Culturales

En las fronteras culturas desarrolla un fenómeno denominado "frontera cultural" se refiere a que dentro de una sociedad existen grupos que son discriminados por distintos motivos como el estatus económico que los margina a vivir en ciertas zonas denominadas "pobres" que por la ubicación existe una falta de servicios básicos como el servicio eléctrico, agua potable, centros de salud, escuelas etc. Lo que va minando su personalidad hasta el extremo de sentirse inferiores además los impide obtener una orientación y educación sexual oportuna estos factores son determinantes más no dictatoriales a hacer personas más propensas a realizar actos contra la libertad sexual de una persona o en este caso un menor de edad. (Lemlij, 2020)

Estas fronteras también son conductuales es por eso que en todos los estratos sociales se presentan estas agresiones sexuales, ya que una buena educación, un estrato social alto etc. no garantiza que la persona pueda llegar a cometer este acto ilegal, ya que lo determinante son los desvíos de personalidad y enfermedades mentales.

3.2.2.2. Factor económico

Según el INEI en el 2019 los niveles de pobreza en el Perú alcanzan al 20.2\% de los peruanos, estas personas tienen un gasto per cápita mensual menor a s/352 soles. Sin embargo los departamentos con mayores índices de pobreza son Ayacucho, Cajamarca, Huancavelica y Puno con niveles del 34.4\% a 39.4\% 


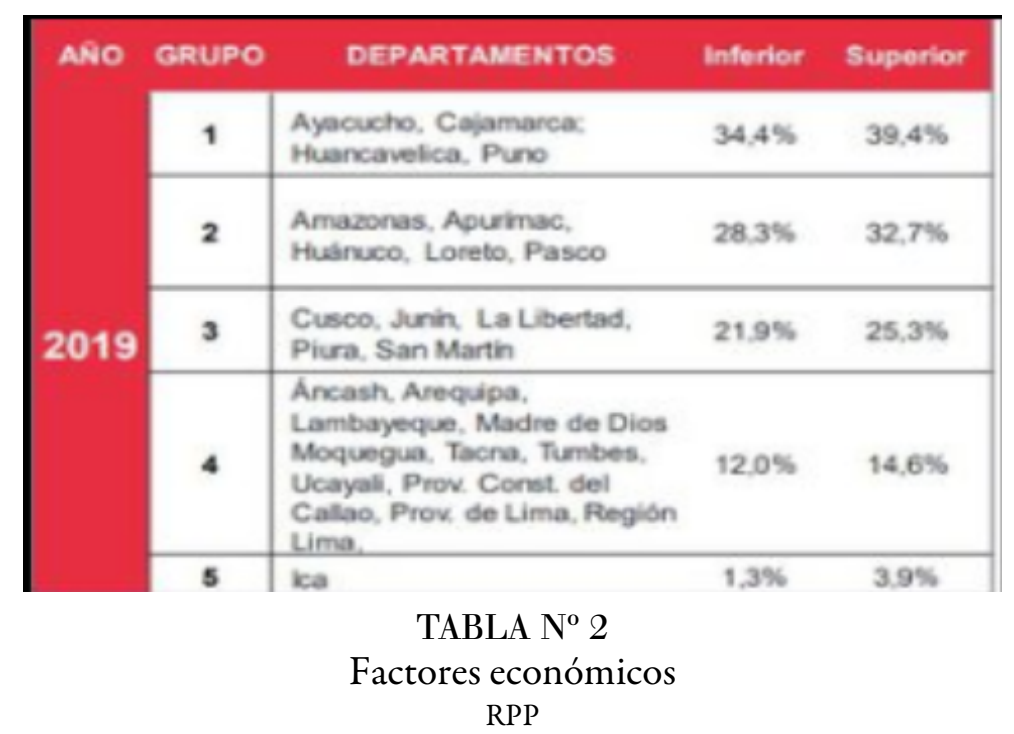

Durante el estado de emergencia debido al confinamiento estos niveles de pobreza aumentaron, justamente en estos lugares donde ya antes de la pandemia existían recursos limitados y poco o nulo acceso a los servicios básicos y de atención esto aumentado al confinamiento anulo cualquier posibilidad de denuncia, es por eso que en estas zonas existen muchos casos pero pocas denuncias como se observa en las estadísticas dadas por MIMP , pero también afecto al aspecto psicológico ya que esta crisis económica llevo a las personas a situaciones críticas debido a los escasos recursos y pérdida de puestos laborales, está comprobado por diversos estudios como el de la Dra. Ana Zavaleta que la falta de dinero genera un gran nivel de estrés al grado de llevar a realizar actos que en una situación "normal” no lo realizarían, la desesperación o hasta la depresión expresados en actos violentos esto se suma a que como lo dijo la ex ministra Gloria Montenegro "El agresor siempre estuvo en casa" después de un análisis grupal pudimos llegar a la conclusión que esta situación límite pudo conducir a muchas personas a convertirse en agresores sexuales como una forma equivoca de liberar tensión o llenar un vacío. (Redacción Gestión, 2020)

\subsection{ANÁLISIS DE LOS FACTORES CRIMINOLÓGICOS DE LA VIOLACIÓN SEXUAL DE} MENORES DESDE LA CRIMINOLOGÍA CLÍNICA

En este sentido, la criminología clínica busca entender por qué se producen los comportamientos delictivos, a fin de poder solucionar el problema subyacente. De esta manera, uno de los principales objetivos de esta disciplina es el de reinsertar a los delincuentes dentro de la sociedad. Teniendo como referencia la criminología clínica establecemos que nos enfocaremos en dos teorías de la conducta criminal estáticas: Teorías de los Rasgos y la Teoría del Autocontrol que nos ayudara a entender el comportamiento del sujeto activo del delito de violación sexual de menores que suscitaron que la consumación de este delito aumentara en el contexto del confinamiento por la pandemia Covid 19.

\subsubsection{TEORÍA DE LOS RASGOS.}

Esta teoría nos señala que, "al estructurar la predisposición biológica de la personalidad, menciona que la dimensión psicoticismo es la que está más atada al comportamiento criminal, aunque sin desligarse de la interacción con la extraversión y el neuroticismo. De este modo, el psicoticismo explicaría la insensibilidad a los demás, la búsqueda de sensaciones y la falta de respeto por el peligro”. (Eysenck \& Gudjonsson, 1989)

El psicoticismo, según Eysenck ésta es una dimensión sobre la vulnerabilidad a conductas impulsivas, agresivas o de baja empatía. Son fríos, egocéntricos e irresponsables, pero también son más creativos, objetivos, realistas, competitivos, originales y críticos.

Estableciendo como principales factores que desencadenaron la consumación del tipo penal de violación de menores de edad son las siguientes: 


\subsubsection{Violación por un miembro del entorno familiar: mecanismo de control}

$\mathrm{Al}$ respecto la Ministra de la Mujer y Poblaciones Vulnerables Antonia Rosario Sasieta Morales, sostiene que el mayor porcentaje de los agresores de los menores de edad se encuentran en sus propias casas que aprovechan el poder de control que tienen sobre ello. Las violaciones son llevadas a cabo con un propósito independiente de la satisfacción sexual y de poder del agresor. Son realizadas con el fin de ejercer poder y superioridad sobre la víctima para que esta no se atreva a denunciarlo y en este contexto de pandemia donde no se tiene "el control de nada" al estar a la merced del estado por el estado emergencia es una forma de sentirse seguro y tener la sensación de control y poder sobre algo o alguien.

Para la ONU informó que, derivado del contagio del Covid-19, aproximadamente 90 países han tomado medidas de aislamiento social, refugiando a 4 mil millones de personas en sus hogares. Lamentablemente para muchas mujeres e infantes, la violencia doméstica se ha recrudecido, ya que su agresor está todo el tiempo en casa y han sido separadas de las personas o recursos que pueden ayudarlas ante una emergencia.

3.3.1.2. Violador en búsqueda de confianza o compensación

Durante el estado de emergencia y confinamiento muchas familias han sido separadas ya sea porque se encontraban en distintas ciudades por motivos de trabajo u otros y se quedaron varadas o por evitar contagiar a miembros de la familia en la situación de "vulnerables" esto hizo que muchos menores se encuentren al cuidado de hermanos mayores, primos, tíos etc., esta situación género que se encontraran solos con su agresor. Este tipo de violador que tiene una percepción distorsionada de la relación entre él y el agredido. El agresor considera que su actuación hará disfrutar a la víctima, ya que el menor separado de círculo familiar de confianza puede presentar algún tipo de depresión, el agresor aprovechando esto y con una percepción errónea comete este acto inclusive piensa que acercará a la persona objeto de su deseo, pudiendo llegar a establecer una relación romántica.

\subsubsection{Violador sádico}

Antes de la pandemia, en muchos hogares existía otro problema grave que aqueja a nuestra sociedad, la violencia familiar, por el confinamiento y al encontrarse impedidos de salir de sus casas, estos episodios se agravaron y se volvieron más continuos, también por la convivencia "obligada ", pasando de agresiones físicas a sexuales en estos casos el inicio de una interacción que el sujeto considera excitante puede provocar que la agresividad del sujeto aumente y experimente impulsos agresivos hacia su víctima, forzándola. No es infrecuente que presenten trastorno antisocial y la parafilia conocida como sadismo sexual, y que en el caso de la violación se expresa de forma directa, sin filtros.

\subsubsection{TEORÍA DEL AUTOCONTROL}

Las diferencias entre los individuos en el delito dependían de una sola construcción teórica subyacente de autocontrol que persiste desde la infancia hasta la edad adulta. Esta teoría argumenta que si las características personales subyacen a los resultados del comportamiento, así como la elección del contexto social, atribuir el poder causal a factores contextuales en la conformación del desarrollo conductual sería falaz. Así, las personas con bajo autocontrol tienen una alta prevalencia, una aparición temprana, una terminación tardía y una larga carrera criminal. (Gottfredson \& Hirschi, 1990)

Estos teóricos argumentan que la criminalidad en cualquier etapa del curso de la vida se debe a estos rasgos estables en el tiempo. En base de esta teoría se estableció que estando en un estado donde la predominancia es el machismo como factor principal para que el agresor aprovechando su ventaja de control sobre la victima pueda ocasionar el daño irreversible como es el caso de una violación sexual y este factor no solo se centra en adultos si no lamentablemente se presenta entre adolescentes que carecen de madurez emocional lo que ocasiona la búsqueda de una satisfacción personal y la compensación del dolor interno de haber sido víctima del mismo tipo sexual que ahora cometen. Cuyos principales factores analizaremos a continuación:

3.3.2.1. Sociedad machista en el acto sexual

Según la encuesta realizada por Pulso Perú en el año 2016 el 74\% de la población considera que el Perú es una sociedad machista. Para el psiquiatra Yuri Cutipé, las personas que cometen estos actos violentos no 
necesariamente tienen una enfermedad mental, sino que el contexto cultural y los valores aprendidos tienen mucho que ver.

El machismo como una "forma de sexismo caracterizada por la prevalencia del varón" y también la califica como la "actitud de prepotencia de los varones respecto de las mujeres". Así, se trata de un prejuicio social que generalmente se aprende en el hogar, donde se asumen como naturales ciertas ideas y actitudes que denigran a la mujer y que se ven reflejadas en la forma de actuar de los padres y en su trato con sus hijos e hijas. (RPP Noticias, 2019)

Esta conducta también como es natural también se traslada al plano de las relaciones sexuales donde pensamientos como "ella tiene que complacerme" "el placer es para el hombre y la mujer tiene que ser quien lo complazca "o frases del vulgar criollo como " tienes que atenderme "refiriéndose a una obligación de la mujer a tener relaciones sexuales cuando el varón lo quiera, también " te fui infiel porque me tenías descuidado" lamentablemente muchas de estas ideas son socialmente aceptadas por lo que el varón desde la pubertad crece con una mentalidad de superioridad y sometimiento de la mujer a su deseo, de igual forma sucede con las mujeres que crecen con este estigma social por lo que lo aceptan como algo "normal".

También la infidelidad es un factor que influye en estas conductas machistas, ya que es aceptado como “ normal " o hasta incluso sinónimo de virilidad, de esta manera el varón o mujer que comete este acto pierde el respeto hacia el género opuesto.

3.3.2.2. Agresores adolecentes por la falta de madurez emocional

Se encontró que los adolescentes abusadores sexuales de menores, en relación con los agresores de iguales, presentan mayores déficits en el funcionamiento psicosocial, mayores alteraciones emocionales de ansiedad y depresión y son más propensos a abusar de familiares que de desconocidos. Según reportes del MIMP un gran porcentaje de victimarios son menores de edad (adolecentes), sumado a trastornos preexistentes y debido a la pandemia los párvulos que se encuentran en formación de su personalidad y madurez sexual, se encontraron encerrados y con una interacción social casi nula esta fundamental para el desarrollo del menor, provocando que experimenten cuadros de depresión, un razonamiento erróneo respecto a los actos de expresión sexual y percepción distorsionada del placer. (Castillero, 2018)

En muchos ya presentaban un rasgo multifactorial de personalidad de parafilia es decir sentir excitación o placer sexual que obtiene con una persona menor de edad, poca educación sexual, también puede desembocar a que el adolescente estando en la posición de hermano, primo, tío etc. Cometa una violación de la libertad sexual del menor (Benedicto et al., 2017)

\subsection{MEDIDAS ADOPTADAS POR EL ESTADO PERUANO EN EL CONTEXTO DE EMERGENCIA}

A inicios del estado de emergencia sanitaria, las disposiciones normativas señaladas en el punto anterior se mantuvieron vigentes para la atención de los casos de violencia contra las niñas, niños y adolescentes, específicamente en los casos de violación sexual, que fueron considerados por todas las instituciones que integran el sistema de justicia como graves y urgentes.

Sin embargo, con la finalidad de garantizar la salud integral de las niñas, niños y adolescentes víctimas de violencia sexual, específicamente, violación sexual, el Ministerio de Salud publicó la Directiva Sanitaria 094-MINSA/2020/DGIESP para garantizar la salud de las gestantes y la continuidad de la atención en la planificación familiar ante la infección por COVID-19, el cual tiene un apartado sobre el kit para la atención de casos de violencia sexual.

El Poder Ejecutivo publicó el Decreto Legislativo 1470 el 27 de abril que estableció medidas para garantizar la atención y protección de las víctimas de violencia contra las mujeres y los integrantes del grupo familiar durante la emergencia sanitaria declarada por el COVID-19, donde están incluidos las niñas, niños y adolescentes víctimas de violencia sexual. Se establecieron disposiciones sobre el derecho a la salud y el procedimiento de riesgo o desprotección familiar, durante la emergencia sanitaria a causa del COVID-19, 
para este grupo incluyendo a las víctimas de todas las modalidades de violencia. Es importante destacar que el procedimiento establecido por el Decreto Legislativo 1470 es más expeditivo por ejemplo:

- La Policía Nacional del Perú y el Ministerio Público tienen que comunicar de inmediato al Poder Judicial para que emita dentro de las 24 horas las medidas de protección, prescindiendo de la audiencia, la ficha de valoración de riesgo y el informe pericial.

- El magistrado o la magistrada solo necesitan la documentación que se le pueda enviar de inmediato, para ello tiene la libertad de llamar a la víctima por cualquier medio tecnológico, para que se le realice preguntas pertinentes sobre su situación de riesgo, teniendo en consideración el confinamiento social.

- Asimismo, dicha norma señala que una medida de protección idónea en estos casos es el retiro del agresor o agresora, y lo que se ha prohibido es la medida de cese de la violencia. En esta norma se ha contemplado el derecho a la salud integral y a la intervención de la Unidad de Protección Especial en los casos donde se evalúe riesgo o desprotección familiar.

3.4.1. Líneas telefónicas de denuncia más directas

Además de la normativa se implementaron líneas de ayuda como la línea 100 que es un servicio telefónico gratuito y especializado cuya finalidad es brindar en forma inmediata y eficaz atención a las víctimas de casos de violencia familiar y sexual, este servicio hasta el 21 de Agosto y según declaraciones de la ministra Antonina Rosario Sasieta Morales atendió más de 113,000 llamadas de las cuales el 76\% son casos de violencia sexual y el $45 \%$ de menores, también se implementó la línea 1810 para atender las denuncias sobre niños, niñas y adolescentes víctimas de violencia física.(Guzmán,2020)

También señalo que el número de casos pueden llegar a ser el doble debido a que estas denuncias pertenecen en un mayor porcentaje a áreas urbanas por lo que los casos de violencia sexual contra menores en zonas rurales no están siendo completamente registrados.

3.4.2. Problemas identificados en la atención de casos de agresión sexual durante el estado de emergencia y después de publicado el DL 1470

Según el informe 007-2020 realizado por la Defensoría del Pueblo nos señala que a pesar de implementado el decreto legislativo 1470 dando las directrices y facilidades para la atención de casos aún se presentan deficiencias que se vuelven obstáculos al momento de atender un caso de agresión sexual de menores, algunos identificados son:

- Vulneración al derecho a la asistencia jurídica y defensa pública:

El derecho a la asistencia jurídica y defensa pública, a través del acompañamiento de un/a abogado/a para la víctima niña, niño o adolescente también ha sido vulnerado después de la publicación del Decreto Legislativo 1470. Se evidencian en dos casos presentados en Cajamarca y La Libertad, la Policía Nacional del Perú una vez más no cumplió con informar a los Centros de Emergencia Mujer o las Oficinas de Defensa Pública para garantizar el derecho a la asistencia jurídica y defensa pública de las víctimas, por lo que la Defensoría del Pueblo fue la que informó, en estos casos, a los Centros de Emergencia de la zona. Mientras que, en un caso en Tacna, el Centro de Emergencia Mujer no brindó sus servicios de asistencia jurídica de manera inmediata, a pesar de que la Policía Nacional del Perú le comunicó el mismo día que recibió la denuncia de la víctima adolescente.

- Vulneración al derecho a la vida, a la integridad personal, al libre desarrollo y a la igualdad:

No informar oportunamente al Poder Judicial ni dictarse las medidas de protección en los tiempos establecidos, causa también una vulneración del derecho a la vida, a la integridad personal, al libre desarrollo y a la igualdad de las víctimas, pues durante ese tiempo la demora hace que las victimas queden en desprotección de los derechos anteriormente mencionados y, además, no se salvaguarda debidamente que no vuelvan a ser objeto de un nuevo acto de violencia por parte del agresor.

Luego de la expedición del Decreto Legislativo 1470, esta situación volvió a ocurrir. En dos casos presentados en Cajamarca y La Libertad, la Policía Nacional del Perú no comunicó de forma inmediata al Poder Judicial sobre los casos de violación sexual denunciados. Mientras que en otros tres casos (Cajamarca, 
La Libertad y San Martín), el Poder Judicial no brindó las medidas de protección respectivas en el plazo establecido por ley. Finalmente, en cuatro casos atendidos (Cajamarca, La Libertad, Puno, San Martín), el Ministerio Público no solicitó medidas coercitivas adecuadas que aseguren la protección de las víctimas, a pesar que los/as agresores/as eran personas cercanas al entorno de la víctima.

\section{CONCLUSIONES}

1. Se evidencia un incremento exponencial alarmante, antes del estado de emergencia con data desde el año 2017 se observaba un crecimiento de casi 600 casos hasta el 2019 por lo cual este delito ya era un problema que necesitaba medidas especiales por parte del Estado, inclusive en los primeros dos meses del 2020 se continuo observando más casos, sumado a que ya existían deficiencias al abordar, por la lentitud de las Instituciones gubernamentales, esto ha empeorado especialmente, en este contexto de emergencia sanitaria por el COVID-19 debido al confinamiento obligatorio durante muchos meses, como indican los reportes del MIMP desde el inicio del estado de emergencia el 15 marzo hasta el mes mayo, se reportaron 226 casos de abuso sexual a menores y hasta el mes de agosto 513 aproximadamente debido a que en zonas rurales existen muchos más casos lamentablemente no reportados.

2. El incremento de este problema social durante la pandemia como lo hemos desarrollado en el presente artículo utilizando la criminología aplicada y clínica nos llevó a concluir que es un problema multifactorial, existía este problema social y año tras año se iba agravando, los aspectos psicológicos ,económicos, sociales y culturales son los principales factores que determinan el crecimiento de este ilícito, todo dentro del contexto de confinamiento conllevo a que la figura de poder ejercida por el agresor creara una situación de vulneración absoluta de la víctima, canalizando toda la frustración(imposibilidad salir o pérdida del trabajo), estrés por la crisis económica, taras psicológicas(abuso que sufrió de niño) y agresividad en el miembro más vulnerable de la familia ,el menor de edad a cargo o en el conocido de la familia más cercano(vecinos). Lo que nos hace reflexionar que la respuesta del estado no solo debe ser represiva castigando con penas privativas de libertad más severas sino que debe ser una atención integral considerando todos estos factores, y preocupándose mucho más por la víctima y la reintegración social de la misma.

3. La respuesta del Estado ha sido ineficiente frente al incremento masivo de casos de violaciones sexuales a menores durante el periodo de estado de emergencia, es verdad que estableció de manera certera líneas de comunicación directa entre las víctimas y las autoridades como la línea 100 pero muchas de estas comunicaciones no han sido atendidas quedando solo en estadísticas, afirmamos que el actuar del estado ha sido lento debido a que durante muchos meses la atención en las instituciones del estado ha sido lenta o hasta incluso inexistente por encontrarse en el proceso de adaptación a los estándares de bioseguridad, el DL 1470 norma que agilizaba y daba más prioridad a la atención de estas agresiones además recién fue publicado y puesto en vigencia 2 meses después de iniciada la emergencia sanitaria y orden de confinamiento. Sin embargo ya una vez puesta en vigencia la normativa se ha observado una ineficiencia en la aplicación de normas por las instituciones encargas como la Policía Nacional del Perú al no comunicar los casos denunciados a los Centros de Emergencia Mujer o las Oficinas de Defensa Pública para garantizar el derecho a la asistencia jurídica y defensa pública de las víctimas, o casos donde la Policía comunico oportunamente pero no se brindó la atención correspondiente, de esta manera vulnerando derechos a menores que se encontraban en la condición de víctimas, cuando estas instituciones debían actuar con más rapidez y eficacia , y no cumpliendo con la política establecida respecto a la atención integral de un caso de violación sexual contra niñas, niños y adolescentes, 
que cuenta con un marco normativo abarcando tres ejes: acceso a la justicia, atención integral en salud y cuidados parentales.

\section{REFERENCIA BIBLIOGRÁFICA}

Benedicto, C., Roncero, D., \& Gonzáles, L. (2017). Agresores sexuales juveniles: tipología y perfil psicosocial es funciónde la edad de sus víctimas. Anuario de Psicología Jurídica 2017, 33, 10.

Castillero, O. (2018). Perfil psicológico del violador: 12 rasgos en común. Psicología y Mente, 5, 4

Cedrón, L. (2020, August 20). Coronavirus en Perú: Estados de ansiedad se incrementan en las personas. El Popular, 1.

Chaparro, N. (2020, March 28). En la pandemia, violencia recrudecida contra la mujer, denuncia Dejusticia. El Espectador, 3.

Defensoria del Pueblo. (2020). PROBLEMÁTICA EN LA ATENCIÓN DE CASOS DE VIOLACIÓN SEXUAL DE NIÑAS, NIÑOS Y ADOLESCENTES EN EL CONTEXTO DE LA EMERGENCIA SANITARIA POR COVID-19.

Eysenck, H., \& Gudjonsson, G. (1989). The causes and cures of criminality. Nueva York: Plenum.

Gottfredson, M., \& Hirschi, T. (1990). A General Theory of Crime. California: Stanford University.

Guzmán, J. C. (2020, August 21). Mimp: Línea 100 atendió más de 113,000 llamadas por violencia familiar durante emergencia. Andina, 1.

Laub, J., \& Sampson, R. (2003). Shared beginnings, divergent lives: Delinquent boys to age. Cambridge: Harvard University.

Lemlij, M. (2020). La necesidad de calmar el vacio y la desesperación durante el confinamiento. America Noticias, Cuarto Poder.

Redacción de la Opinión. (2020, May 16). Violencia de género, la consecuencia invisible de la pandemia. Opinión Diario de Circulación Nacional, 3.

Redacción Gestión. (2020, May 20). Pobreza en Perú bajó el 2019 pero subió el nivel de la pobreza extrema. Gestión, 4. RPP Noticias. (2019, January 16). Machismo: el problema social detrás de la violencia de género. 3.

Sampson, R., \& Laub, J. (1993). Crime in the making:Pathways and turning points through life. Cambridge: Harvard University.

Sociedad LR. (2020, May 8). 226 abusos sexuales a menores de edad se han reportado desde el inicio de la pandemia. La República, 2.

Thornberry, T., \& Krohn, M. (2001). The development of delinquiency: An interactional perspective. En S. White (ed.). Handbook of Youth and Justice, 289-305.

Zabaleta, A. (2016). El dinero como causa de estrés. Innatia.

\section{INFORMACIÓN ADICIONAL}

Investigación formativa realizada por: Estudiantes del curso de Criminología de la Escuela Profesional de Derecho de la Facultad de Ciencias Jurídicas y Políticas de la Universidad Nacional del Altiplano Puno. 